\title{
Author Correction: Reconfigurable beam system for non-line-of-sight free-space optical communication
}

Zizheng Cao, Xuebing Zhang, Gerwin Osnabrugge, Juhao Li, Ivo M. Vellekoop (1) and Antonius M. J. Koonen

Addendum to: Light: Science \& Applications https://doi.org/10.1038/s41377-019-0177-3

Addendum:

We were recently made aware of the fact that Professor Nabeel A. Riza's laboratory theoretically proposed earlier a Non-Line of Sight (NLOS) indoor optical wireless communications arrangement with threedimensional optical beamforming via a spatial light modulator using diffuse reflections from diffuse optical elements ${ }^{1}$. This is exactly the type of arrangement that would benefit greatly from our technique, since the coherent arrayed optical transmitter (CAO-Tx) we proposed makes the reflection focus onto the receiver, rather than diffusely scatter it in all directions. We apologize for the omission of this prior-art.

Published online: 01 July 2021

\section{Reference}

1. Marraccini, P. J. \& Riza, N. A. Smart multiple-mode indoor optical wireless design and multimode light source smart energy-efficient links. SPIE. Digital Library. Opt. Eng. 52, 055001 (2013). 